\title{
Diagnosis of an Inverter IGBT Open-circuit Fault by Hilbert-Huang Transform Application
}

\author{
Bilal Djamal Eddine Cherif ${ }^{1 *}$, Azeddine Bendiabdellah ${ }^{1}$, Mostefa Tabbakh $^{2}$ \\ ${ }^{1}$ Diagnosis Group, Laboratory LDEE, Electrical Engineering Faculty, University of Sciences and Technology of Oran, Oran \\ 31000, Algeria \\ ${ }^{2}$ Department of Electrical Engineering, University of M'sila, M'sila 28000, Algeria
}

Corresponding Author Email: cherif.doc84@gmail.com

https://doi.org/10.18280/ts.360201

Received: 12 January 2019

Accepted: 29 March 2019

\section{Keywords:}

inverter, IGBT, open-circuit, HHT, EMD, CEEMDAN, IMF, spectral envelope, $R M S$

\begin{abstract}
The open-circuit fault of an inverter IGBT switch leads to total or partial loss of control of the phase currents resulting in the dysfunction of the system. Moreover, if the fault is not detected and compensated quickly, it can cause complete shutdown of the system. To ensure the system service continuity, efficient and fast techniques for detecting and locating the open-circuit fault of the IGBT must be implemented. This paper proposes a Hilbert-Huang Transform (HHT) based on the detection of the IGBT open-circuit fault. The proposed technique is based on the complete empirical mode decomposition with adaptive noise (CEEMDAN). This mode is applied to the motor stator current signals to obtain a function called the intrinsic mode function (IMF). The IMF contains the frequency (and its multiples) related to the frequency of the harmonic characterizing the IGBT switch open-circuit fault of the inverter. In order to test the effectiveness of the proposed technique and validate the results, several experimental tests are performed using a test bench.
\end{abstract}

\section{INTRODUCTION}

In general, maintenance is intended to ensure the maximum availability of production equipment at an optimal cost under good conditions of quality and safety. The general principle of the diagnostic algorithms is based on the use of the data recorded on the system and the knowledge that one possesses of its healthy operation (for the detection) or its faulty operation (for the location). These algorithms develop symptoms that reveal the faulty behavior and the nature of the dysfunction. In this framework, static converters, particularly inverters, are mainly present in variable speed electrical drive systems. Reliability data; from the literature; justify the envisaged scope for the implementation of fault tolerance or failure. Figure 1 shows the distribution of faults in $\%$ in an inverter [1].
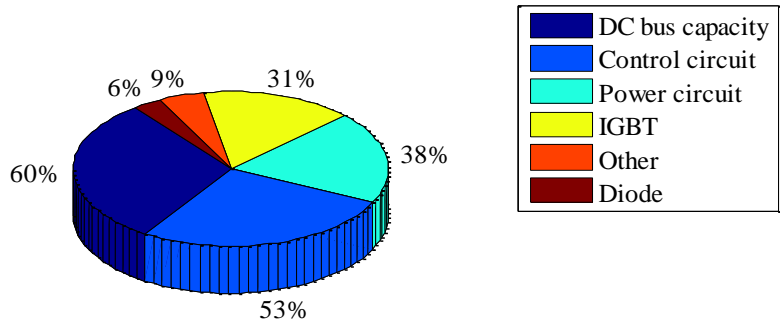

Figure 1. Distribution of faults in $\%$ in a static converter

Among these diagnostic methods there are spectral analysis techniques based on the Fourier transform (FT). The FT provides a good description of the stationary and pseudostationary signals but has many limitations when the signals to be analyzed are not stationary. In this case, the solution would be to use the so-called time-frequency analysis tools. These methods include: the STFT and the Hilbert-Huang Transform (HHT) [2].

The authors Hilbert and Huang have recently proposed a technique that approaches in another angle the problematic of non-stationary signal analysis with the empirical modal decomposition (EMD) approach. The EMD adaptively decomposes a signal in a sum of oscillating components. Unlike FT or wavelets, the basis of the EMD decomposition is intrinsic to the signal. One of the motivations for the development of the EMD is the estimation of the instantaneous frequency (IF) of the signal. Indeed, the conventional approach of estimating the IF based on the Hilbert transformation (HT) is strictly limited to singlecomponent signals. Thus, constraints are imposed on these oscillating components to correctly estimate the IF (with a physical sense) specific to each component present in the signal. The EMD combined with the HT or another method of estimating the IF results in a time-frequency representation (TFR). The EMD is defined by a process called sifting, which decomposes the signal into basic contributions called empirical modes or intrinsic mode functions (IMF). These are signals of amplitude modulation - frequency modulation type mono-component (in broad sense) each of zero average. The principle of the EMD is based on an adapted decomposition describing the signal locally as a succession of contributions of fast oscillations (high frequencies) on slower oscillations (low frequencies) [3-6].

Several papers have been published in this diagnostic field based on the HHT. The author in the paper [7] presents a method using the spectral envelope of the stator current for the online automatic detection of broken bar faults. In this paper, the HHT is used to estimate the severity of faults for 
different loads using classification techniques. The spectral envelope of the stator current makes it possible to read the frequency relative to the fault, which confirms the existence of the fault. The author [8] proposes a method based on the complete empirical ensemble mode decomposition with adaptive noise (CEEMDAN) associated with an optimized Thresholding operation. The CEEMDAN is first applied to the vibration signals to obtain a series of functions called the IMF functions. An approach based on the energy content of each mode with the white noise characteristic is then proposed to determine the trigger point to select the relevant modes. The author in paper [9] presents a rolling fault diagnosis method based on an improvement time-time of Hilbert (HTT a derivative of the HHT) with the main component which is the Denoising HTT transform matrix. The HTT was performed on vibration signals to deduce the transformed matrix. The main component is then used to attenuate the noise of the HHT matrix in order to improve its robustness and extract information and characteristics of the bearing fault.

This paper proposes an HHT-based diagnostic method for detecting the open-circuit fault of an IGBT in an inverter. The proposed technique is based on complete ensemble empirical mode decomposition with an Adaptive Noise (CEEMDAN). This mode is applied to the motor stator current signals to obtain a function called the intrinsic mode function (IMF) containing the frequency of the harmonic relative to the harmonic characterizing the IGBT fault. In order to test the effectiveness of the proposed technique and validate the results, several experimental tests are carried out on the system using a practical test bench at our LDEE laboratory, consisting of an induction motor powered by a two-level three-phase faulty voltage inverter controlled by the MLI-SVM strategy.

\section{HILBERT-HUANG TRANSFORM}

In this section, the principle of HHT will be presented as well as the different versions of decomposition in empirical modes (EMD and CEEMDAN) in addition to the spectral envelope and RMS.

\subsection{EMD algorithm}

The EMD method decomposes the signal into a finite number of IMFs and a residue. It should satisfy the following conditions [10]:

1) The number of extrema and the number of zerocrossings are equal or differ by one.

2) The mean value of the envelopes defined by local maxima and local minima is zero.

For a given signal $(t)$, the EMD algorithm is described in the following steps [10]:

$\mathbf{1}^{\text {st }}$ step: Initialize: $r_{0}=(x(t))$ and $i=1$

$2^{\text {nd }}$ step: Extract the $i I M F$.

(a) Initialize $h_{i(k-1)}=r_{i}, k=1$.

(b) Extract the local Max and Min of $h_{i(k-1)}$.

(c) Interpolate the local Max and Min with cubic spleen lines to form the upper and lower envelopes of $h_{i(k-1)}$.

(d) Calculate the average $m_{i(k-1)}$ of the upper and lower envelopes of $h_{i(k-1)}$.

(e) Let $h_{i k}=h_{i(k-1)^{-}} m_{i(k-1)}$. (f) If $h_{i k}$ is an IMF, set $I M F_{i}=h_{i k}$, otherwise go to step (b) with $k=k+1$.

$3^{\text {rd }}$ step: Define $r_{i+1}=r_{i}-I M F_{i}$

$4^{\text {th }}$ step: Continue the process until the final residue $r_{n}$ satisfies the predefined stopping criterion. The stopping condition $(S D)$ is calculated from the two consecutive sifting results, namely $h_{k-1}$ and $h_{k}$ as [9]:

$$
S D(i)=\sum_{t=0}^{T} \frac{\left|h_{j, i-1}(t)-h_{j, i}(t)\right|^{2}}{\left(h_{j, i-1}(t)\right)^{2}}
$$

where: $T$ is the time duration. The sifting process is terminated when the $S D$ value is greater than a certain threshold. Here a typical value of $S D$ can be set between 0.2 and 0.3 [9].

The signal can be expressed as follows:

$$
x(t)=\sum_{i=1}^{n} c_{i}+r_{n}
$$

\subsection{CEEMDAN algorithm}

The Complete Empirical Ensemble Mode Decomposition an Adaptive Noise (CEEMDAN) is used to solve the EEMD problem related to residual noise and also to the existence of modes with different numbers. The CEEMDAN algorithm is illustrated by the following steps [11]

$1^{\text {st }}$ step: Use the EMD to decompose $I$ realizations of $x+$ $\varepsilon_{0} \omega^{i}(i=1, \ldots \ldots, I)$ in order to obtain its first modes and to calculate the first mode of the CEEMDAN as follows:

$$
\overline{I M F_{1}}=\frac{1}{I} \sum_{i=1}^{I} E_{1}\left(x+\zeta \omega_{i}\right)
$$

With $x, \omega_{i}$ : Gaussian white noise with $\mathrm{N}(0,1), \varepsilon$ : a noise standard deviation, $I$ : Number of sets.

$2^{\text {nd }}$ step: Calculate the first residue $r_{1}=x-\overline{I M F_{1}}$

$3^{\text {rd }}$ step: Use the EMD to decompose $r_{1}+\varepsilon_{1} E_{1}\left(\omega^{i}\right),(i=$ $1, \ldots \ldots, I)$ to get its first modes and define the second mode of CEEMDAN as:

$$
\overline{I M F_{2}}=\frac{1}{I} \sum_{i=1}^{I} E_{1}\left(r_{1}+\zeta_{1} E_{1}\left(\omega^{i}\right)\right)
$$

$4^{\text {th }}$ step: For $k=2 \ldots, k$ the residue is given as follows:

$$
r_{k}=r_{k-1}-\overline{I M F_{k}}
$$

$5^{\text {th }}$ step: Use EMD to decompose the realizations $r_{k}+$ $\varepsilon_{k} E_{k}\left(\omega^{i}\right),(i=1, \ldots \ldots, I)$ and define the $(k+$ 1) ${ }^{\text {th }}$ CEEMDAN mode as follows:

$$
\overline{I M F_{k+1}}=\frac{1}{I} \sum_{i=1}^{I} E_{1}\left(r_{k}+\zeta_{k} E_{k}\left(\omega^{i}\right)\right)
$$

With $E_{k}():. k^{\text {th }}$ IMF product to obtain par the EMD. $6^{\text {th }}$ step: Go to step 4 for the next $k$. 
$7^{\text {th }}$ step: Iterate steps 4-6 until the resulting residue can no longer be decomposed by the EMD. The final residue is given as follows:

$$
r_{n}=x-\sum_{i=1}^{n} \overline{I M F_{i}}
$$

So that the given signal can be expressed by:

$$
x=r_{n}+\sum_{i=1}^{n} \overline{I M F_{i}}
$$

With: $n$ : The total number of modes, $\varepsilon_{k}$ : The amplitude of the added white noise, $\omega$ : White noise with the unit variance.

In this paper, the proposed technique is represented by the flowchart of Figure 2 as follows:

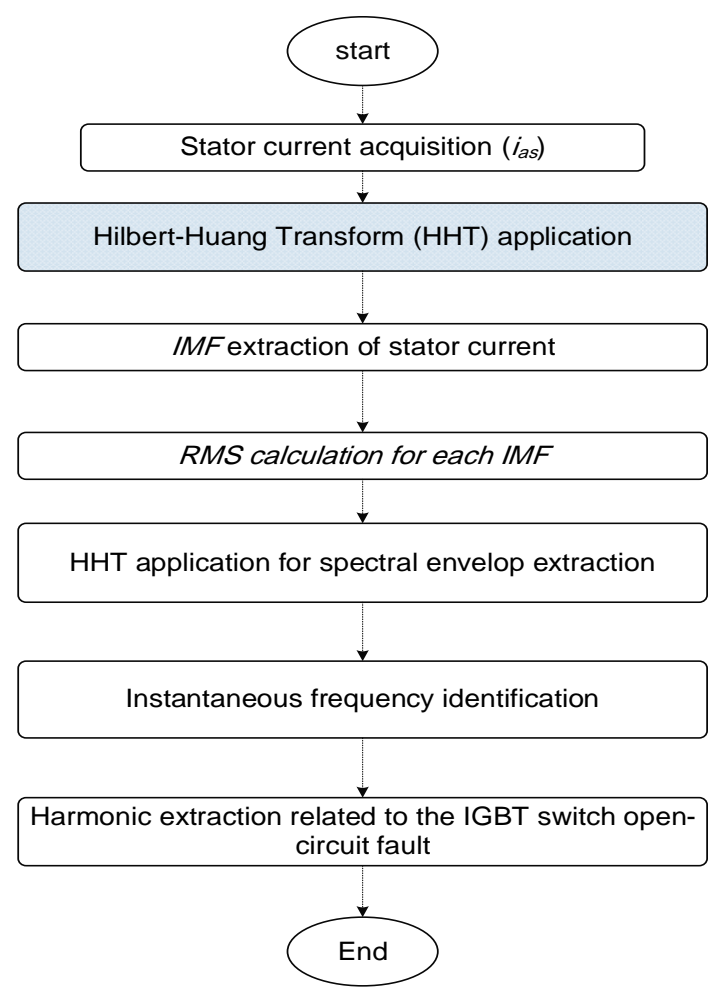

Figure 2. Organizational chart of the proposed method

\section{EXPERIMENTAL \\ INTERPRETATION}

RESULTS

AND

The three-phase inverter used is this work is an IGBTbased three-phase (SEMI-KRON) controlled by the DSPACE 1104 Card. The inverter IGBTs are controlled by the MLISVM strategy. The motor used is of a three-phase squirrel cage type; with a nominal power of $3 \mathrm{Kw}$, a frequency of 50 $\mathrm{Hz}$ and a nominal rotor speed of $1440 \mathrm{rpm}$.

This motor is mechanically coupled to a DC generator used as a load. The measuring system has three voltage sensors (TEKTRONIX P5200) and three Hall-Effect current sensors (FLUCK i30s (AC/DC CURRENT CLAMP)), a tachometer (ONO SOKKI HT-341) and an acquisition card (NI-6330). Finally, the whole set is connected to a computer for visualizing the processed acquired signals as shown in the photo of Figure 3 [12].

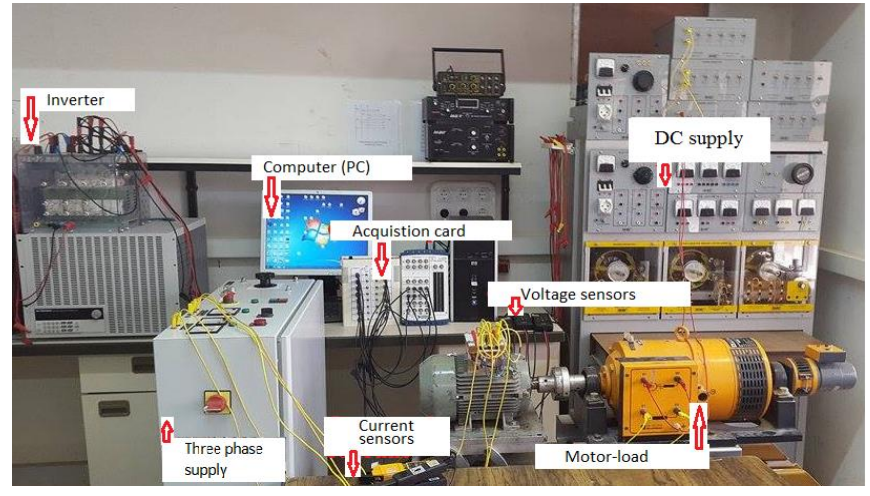

Figure 3. Photo of experimental test-rig [12]

Table 1 presents the induction motor parameters and specifications.

Table 1. Parameters of the induction motor

\begin{tabular}{cc}
\hline Rated Power & $3 \mathrm{KW}$ \\
\hline Supply frequency & $50 \mathrm{~Hz}$ \\
\hline Rated voltage & $380 \mathrm{~V}$ \\
\hline Rated current & $7 \mathrm{~A}$ \\
\hline Rotor speed & $1410 \mathrm{rev} / \mathrm{min}$ \\
\hline Number of rotor bars & 28 \\
\hline Number of stator slots & 36 \\
\hline Power factor & 0.83 \\
\hline Number of pair of poles & 2 \\
\hline
\end{tabular}

Figure 4 shows the structure of the two-level three-phase voltage inverter. The system consists of a three-phase voltage inverter with two levels based on faulty IGBT switches supplying an induction machine.

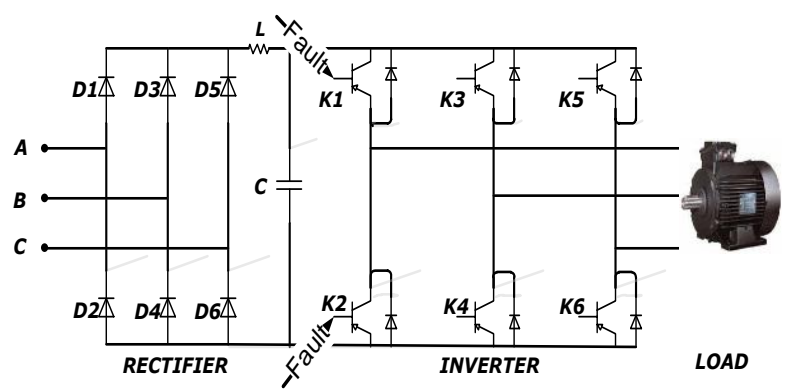

Figure 4. Structure of the converter-motor assembly with open-circuit fault

All the acquisitions were made in nominal mode over a period of 5 seconds with a sampling frequency of $1.5 \mathrm{kHz}$.

The various modes of operation of an inverter-motor assembly made to validate the diagnostic procedure are:

- Operation with a healthy inverter.

- Operation with an inverter open-circuit fault of IGBT $K_{1}$.

- Operation with an inverter open-circuit fault of IGBT $K_{2}$

Figure 5 shows the stator current $i_{a s}$ in both the healthy and open-circuit faulty cases.

Figure 5 shows the stator current in the normal and abnormal operation of the system. The stator current is characterized with respect to the normal regime by a sudden variation at the instant of the application of the open-circuit fault at the $K_{l}$ switch resulting in a loss of the positive half- 
cycle of the current. On the other hand, in the case of a fault at the switch $K_{2}$, a loss of the negative alternation is observed.
Figure 6 depicts the selected IMFs in the healthy and the open-circuit faulty cases.

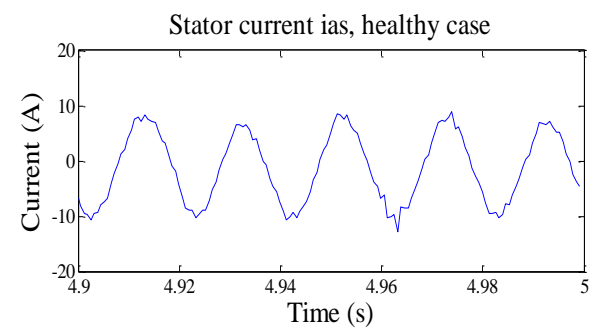

(a) Healthy state

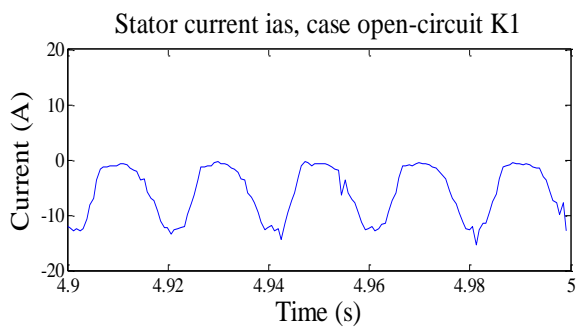

(b) Open-circuit at switch $K_{1}$

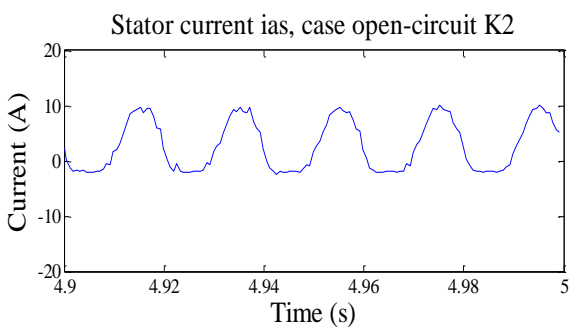

(c) Open-circuit at switch $K_{2}$

Figure 5. Stator current
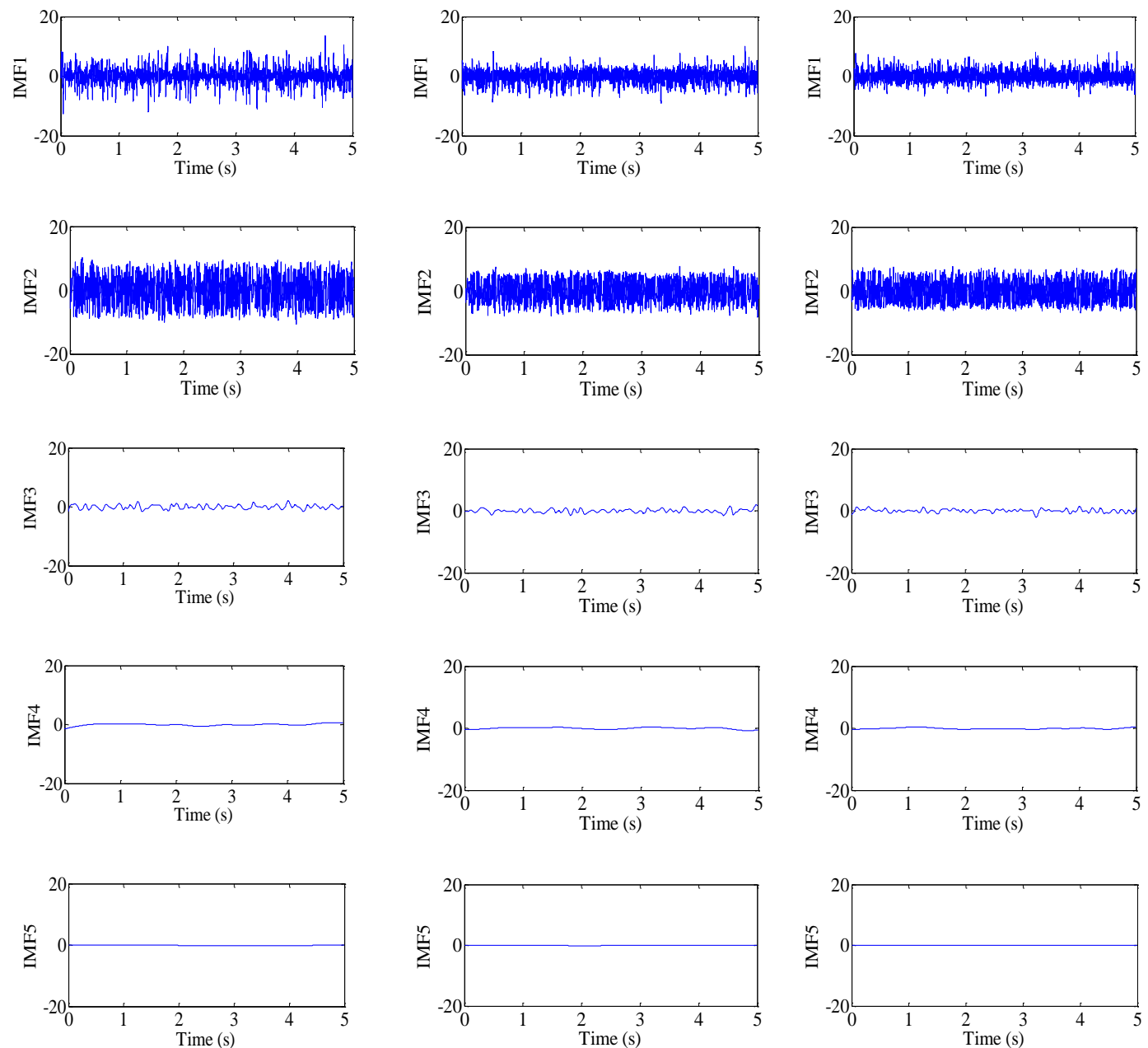

(a) Healthy state

(b) Open-circuit at switch $K_{1}$

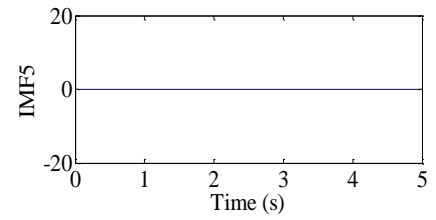

(c) Open-circuit at switch $K_{2}$

Figure 6. IMF

\subsection{Statistical study}

RMS: it is a very characteristic value of the signal, since it has a direct relation with the energy contained in it:

$$
R M S=\sqrt{\frac{1}{T} \int_{0}^{t} I M F^{2}(t) d t}
$$

where: IMF $(t)$ is the representative function of the signal and " $t$ " is the analysis time.

Table 2 presents the RMS value of each IMF. After analyzing the results obtained in Table 2 for each IMF we observed logic in $\mathrm{IMF}_{1}$ that identifies the IGBT fault. $K_{1,3,5}$ are always lower than the values $K_{2,4,6}$ respectively in the case of an open-circuit fault. The IMF 1 signal is therefore the one to be used to detect and locate the harmonics that characterizes the open-circuit fault IGBT. 
Table 2. RMS value of each IMF

\begin{tabular}{cccccc}
\hline State & IMF $_{\mathbf{1}}$ & IMF $_{\mathbf{2}}$ & IMF $_{\mathbf{3}}$ & IMF $_{\mathbf{4}}$ & IMF $_{\mathbf{5}}$ \\
\hline Heathy & 82.7486 & 251.5579 & 392.6519 & 93.3259 & 54.5970 \\
\hline Open $K_{1}$ & 66.5023 & 188.9364 & 296.1187 & 65.0230 & 44.5439 \\
\hline Open $K_{2}$ & 67.2751 & 166.4776 & 292.3342 & 66.4481 & 37.1695 \\
\hline Open $K_{3}$ & 95.8056 & 299.4912 & 478.9089 & 104.0427 & 70.1447 \\
\hline Open $K_{4}$ & 90.9676 & 290.8385 & 463.9138 & 103.9608 & 55.5179 \\
\hline Open $K_{5}$ & 85.4351 & 251.0758 & 439.7275 & 87.3736 & 56.3215 \\
\hline Open $K_{6}$ & 85.5871 & 270.7648 & 421.8767 & 96.9308 & 55.5287 \\
\hline \multicolumn{5}{c}{ Stator current $\boldsymbol{i b s}$} \\
\hline Heathy & 78.1394 & 224.4353 & 400.2280 & 84.5102 & 51.3490 \\
\hline Open $K_{1}$ & 83.8776 & 279.4305 & 428.2569 & 98.4593 & 59.4270 \\
\hline Open $K_{2}$ & 80.4376 & 259.0379 & 434.0585 & 95.5906 & 63.0193 \\
\hline Open $K_{3}$ & 62.0850 & 176.8302 & 293.7399 & 64.8789 & 47.8038 \\
\hline Open $K_{4}$ & 64.0776 & 180.4871 & 301.9739 & 66.5300 & 41.6790 \\
\hline Open $K_{5}$ & 92.9372 & 276.8886 & 495.3335 & 116.2171 & 68.1189 \\
\hline Open $K_{6}$ & 93.9432 & 282.5435 & 474.9952 & 103.5992 & 62.0288 \\
\hline \multicolumn{5}{c}{ Stator current ics } \\
\hline Heathy & 80.5750 & 257.3659 & 422.6741 & 99.5111 & 60.7217 \\
\hline Open $K_{1}$ & 94.2862 & 289.2505 & 485.5097 & 102.1549 & 59.3271 \\
\hline Open $K_{2}$ & 91.8464 & 312.2062 & 478.6916 & 103.9096 & 69.4545 \\
\hline Open $K_{3}$ & 82.6258 & 282.0376 & 428.4229 & 102.7446 & 54.2908 \\
\hline Open $K_{4}$ & 79.8416 & 262.4210 & 422.2279 & 103.5813 & 56.0183 \\
\hline Open $K_{5}$ & 62.3196 & 194.9059 & 297.3967 & 63.2087 & 43.7761 \\
\hline Open $K_{6}$ & 65.1510 & 181.7017 & 304.8296 & 68.6557 & 42.2992 \\
\hline
\end{tabular}

\subsection{Hilbert spectral envelope}

The characteristics of the Hilbert spectral envelope are quoted as follows:

(a) Elimination of the fundamental $(50 \mathrm{~Hz})$ of the current spectrum.

(b) Shifting of all frequency signatures to the left of $50 \mathrm{~Hz}$.

(c) Visibility of the frequency signatures of the faults those are generally of very low amplitude due to the absence of the fundamental.

(d) Visibility of the frequency signatures of faults allowing the use of the linear scale instead of the semi-logarithmic scale.

(e) Elimination of the fundamental; only one characteristic frequency component of the fault appears instead of the three lateral bands multiple of 2 . As for example for the signature of the open-circuit fault of an IGBT of the inverter.

Figure 7 shows the IMF spectral envelope in the healthy case and the case of open-circuit fault at the IGBT switches $K_{1}$ and $K_{2}$.

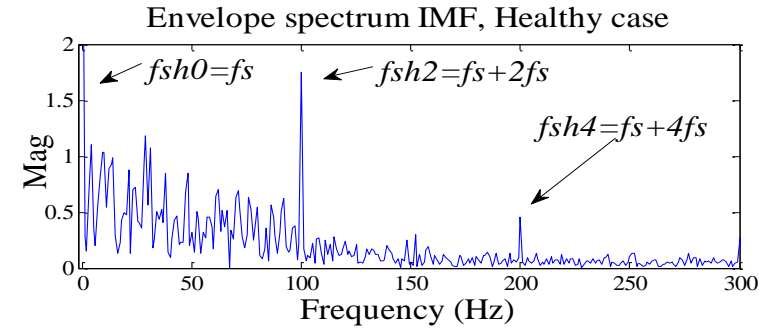

(a) Healthy state

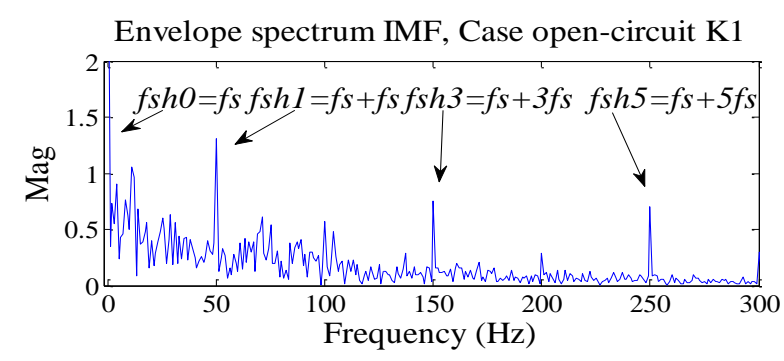

(b) Open-circuit at switch $K_{1}$

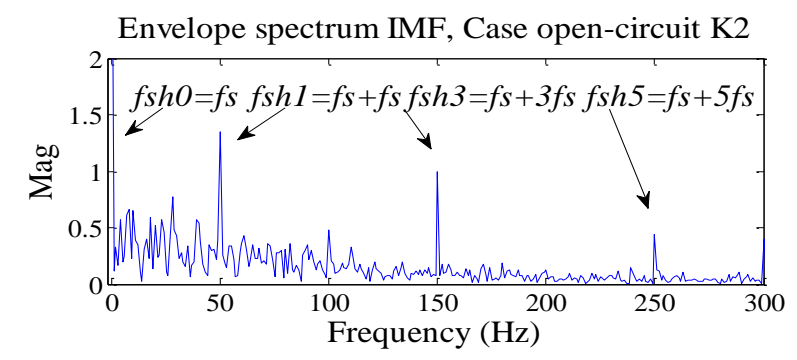

(c) Open-circuit at swith $K_{2}$

Figure 7. Spectral envelope

Figure 7(a), the harmonic $f_{s}$ is no more visible because of the Hilbert spectral envelope effect that causes the elimination of this harmonic and the shift of all frequencies to the left of the harmonic of $50 \mathrm{~Hz}$. This explains the existence of the harmonics $\left(f_{s h 2}\right)$ and $\left(f_{s h 4}\right)$.

In the case of the open-circuit fault of the IGBT switches at $K_{1}$ and $K_{2}$, depict the existence of the fundamental harmonic $f_{s}(50 \mathrm{~Hz})$ and other harmonics $2 f_{s}(100 \mathrm{~Hz}), 3 f_{s}$ $(150 \mathrm{~Hz})$ and $4 f_{s}(200 \mathrm{~Hz})$. This explains the existence of the harmonic $\left(f_{s h 1}\right),\left(f_{s h 3}\right)$ and $\left(f_{s h 5}\right)$ in the Hilbert spectral envelope shown in Figure 7(b) and 7(c) hence replacing the harmonic $\left(f_{s}+f_{s}\right),\left(f_{s}+3 f_{s}\right)$ and $\left(f_{s}+5 f_{s}\right)$ with a shifting of $50 \mathrm{~Hz}$.

Table 3 summarizes the amplitudes of the harmonics $\left(f_{s h 1}\right.$, $f_{s h 2,} f_{s h 3,}, f_{s h 4}$ and $\left.f_{s h 5}\right)$ of the spectral envelope in the healthy and the IGBT open-circuit faulty cases.

Table 3. Amplitude of the $\left(f_{s h 1}, f_{s h 2,}, f_{s h 3}, f_{s h 4}\right.$ and $\left.f_{s h 5}\right)$ of spectral envelope

\begin{tabular}{ccccccc}
\hline Harmonics & $\boldsymbol{f}_{\boldsymbol{s h} \mathbf{1}}(\mathbf{d B})$ & $\boldsymbol{f}_{\boldsymbol{s h} \mathbf{2}}(\mathbf{d B})$ & $\boldsymbol{f}_{\boldsymbol{s h} \mathbf{3}}(\mathbf{d B})$ & $\boldsymbol{f}_{\boldsymbol{s h} \mathbf{4}}(\mathbf{d B})$ & $\boldsymbol{f}_{\boldsymbol{s h} \mathbf{5}}(\mathbf{d B})$ & $\boldsymbol{f}_{\text {inst }}(\mathbf{H z})$ \\
\hline Healthy case & 0 & 1.746 & 0 & 0.4605 & 0 & 0 \\
\hline Open $\mathrm{K}_{1}$ & 1.309 & 0 & 0.7544 & 0 & 0.707 & 50 \\
\hline Open $\mathrm{K}_{2}$ & 1.344 & 0 & 0.9951 & 0 & 0.4363 & 50 \\
\hline Open $\mathrm{K}_{3}$ & 0.8918 & 0 & 0.8079 & 0 & 0.4424 & 50 \\
\hline Open $\mathrm{K}_{4}$ & 1.329 & 0 & 0.7949 & 0 & 0.7329 & 50 \\
\hline Open $\mathrm{K}_{5}$ & 1.268 & 0 & 1.04 & 0 & 0.5924 & 50 \\
\hline Open $\mathrm{K}_{6}$ & 1.316 & 0 & 0.7719 & 0 & 0.615 & 50 \\
\hline
\end{tabular}

According to Table 3, a comparative analysis between the healthy and the IGBT open-circuit fault cases clearly shows a frequency signature at about $(50 \mathrm{~Hz})$ for the Hilbert spectral envelope. It should be noted that this frequency is the one that characterizes the open-circuit fault of the IGBT. In order to confirm the validity of this observation, the instantaneous Hilbert frequency is identified and shown in Figure 8. 


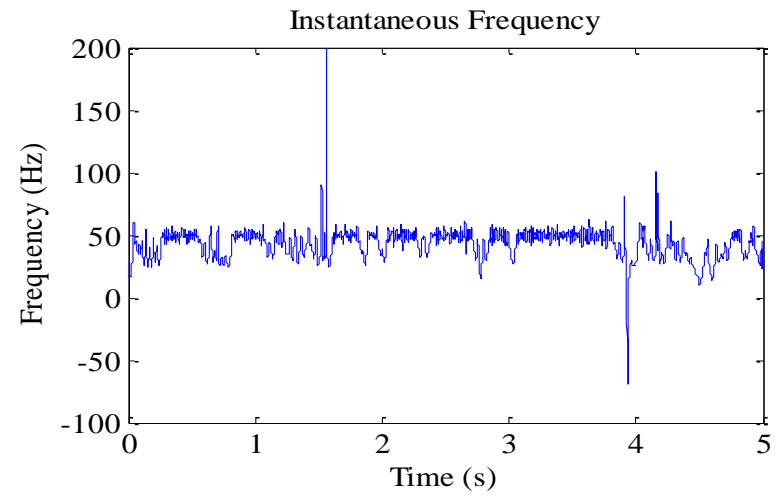

Figure 8. Instantaneous frequency of HHT in case of opencircuit fault of switches at $K_{1}$ and $K_{2}$

Figure 8 shows that the instantaneous frequency $\left(f_{\text {sh }}=50\right.$ $\mathrm{Hz})$ is the frequency that characterizes the open-circuit fault of the IGBT.

\section{CONCLUSIONS}

In this paper, a method for diagnosing and detecting the harmonic characteristic of the open-circuit fault of an IGBT of the two-stage three-phase inverter supplying an induction motor is proposed. This diagnostic method is based on the Hilbert-Huang transform to identify the instantaneous frequency that allows us to detect the frequency characterizing the open-circuit fault of the IGBT. This paper study is based on the extraction of the IMF for the healthy and the IGBT open-circuit fault cases by using the algorithm (CEEMDAN). To detect the open-circuit faults related to the resulting IMF, the Hilbert spectral envelope are conducted to identify the instantaneous frequency. This instantaneous frequency is the frequency characterizing the open-circuit fault of the IGBT. The method proposed is more efficient and more sensitive to the early detection and the diagnosis of open-circuit fault of the IGBTs of the inverter when compared to the conventional methods for example the wavelet or the STFT. The various results obtained are validated by several experimental works carried out in the LDEE laboratory by the diagnostic group to assess the effectiveness and the merits of the proposed HHT approach.

\section{REFERENCES}

[1] Kakurin, A.M., Orlovsky, I.I. (2005). Hilbert-Huang transform in MHD plasma diagnostics. Plasma Physics Reports, 31(12): 1054-1063. https://doi.org/10.1134/1.2147651

[2] Neto, E.P.S., Custaud, M.A., Cejka, J.C., Abry, P., Frutoso, J., Gharib, C., Flandrin, P. (2004). Assessment of cardiovascular autonomic control by the empirical mode decomposition. Methods of Information in
Medicine,

43(1):

$60-65$.

https://doi.org/10.1267/METH04010060

[3] Janosi, I.M., Muller, R. (2005). Empirical mode decomposition and correlation properties of long daily ozone records. Physical Review E, 71, 056126. https://doi.org/10.1103/PhysRevE.71.056126

[4] Kerschen, G., Vakakis, A.F., Lee, Y.S., McFarland, D.M., Bergman, L.A. (2006). Toward a fundamental understanding of the Hilbert-Huang transform in nonlinear structural dynamics. Proceedings of the 24th International Modal Analysis Conference (IMAC), StLouis.

[5] Khelif, A.M., Cherif, B.D.E., Bendiabdellah, A. (2018). Diagnosis of SVM controlled three-phase rectifier using mean value of park currents technique. International Review on Modelling and Simulations (IREMOS), 11(2): 93-101. https://doi.org/10.15866/iremos.v11i2.13848

[6] Zhang, J.H., Luo, H., Zhao, J., Wu, F. (2015). A fuzzybased approach for open-transistor fault diagnosis in voltage-source inverter induction motor drives. The European Physical Journal Applied Physics, 69(2). https://doi.org/10.1051/epjap/2015140066

[7] Raj, N., Mathew, J., Jagadanand, G., George, S. (2016). Open-transistor fault detection and diagnosis based on current trajectory in a two-level voltage source inverter. Procedia Technology, 25: 669-675. https://doi.org/10.1016/j.protcy.2016.08.159

[8] Ouanas, A., Medoued, A., Salim, H., Mordjaoui, M., Sayad, D. (2018). Automatic and online detection of rotor fault state. International Journal of Renewable Energy Development, 7(1): 43-52. https://doi.org/10.14710/ijred.7.1.43-52

[9] Rabah, A., Abdelhafid, K., Azeddine, B., Ziane, D. (2018). Rolling bearing fault diagnosis based on an improved denoising method using the complete ensemble empirical mode decomposition and the optimized thresholding operation. IEEE Sensors Journal, 18(17): 7166-7172. https://doi.org/10.1109/JSEN.2018.2853136

[10] Wu, Z.H., Huang, N. (2011). Ensemble empirical mode decomposition: A noise-assisted data analysis method. Advances in Adaptive Data Analysis, 1(1): 385-388. https://doi.org/10.1142/S1793536909000047

[11] Xue, X.M., Zhou, J.Z., Xu, Y.H., Zhu, W.L., Li, C.S. (2015). An adaptively fast ensemble empirical mode decomposition method and its applications to rolling element bearing fault diagnosis. Mechanical Systems and Signal Processing, 62-63: 444-459. https://doi.org/10.1016/j.ymssp.2015.03.002

[12] Cherif, B.D.E., Bendiabdellah, A. (2018). Detection of two-level inverter open-circuit fault using a combined DWT-NN approach. Journal of Control Science and Engineering (JCSE), Volume 2018. https://doi.org/10.1155/2018/1976836 